\title{
Correspondence \\ Working Mechanism Underlying the Reduction of the Behavioral and Accumbal Dopamine Response to Cocaine by $\alpha$-I-Adrenoceptor Antagonists
}

\author{
Michel MM Verheij*,', Tadashi Saigusa ${ }^{2}$, Noriaki Koshikawa ${ }^{2}$ and Alexander R Cools' \\ 'Department of Cognitive Neuroscience, Division of Psychoneuropharmacology, Donders Institute for Brain, Cognition and Behaviour, Radboud \\ University Nijmegen Medical Centre, Nijmegen, The Netherlands; '2Department of Pharmacology, Nihon University School of Dentistry, Tokyo, \\ Japan
}

Neuropsychopharmacology (2013) 38, 540-54I; doi: I0.1038/npp.2012.209

In the late 1970s, it was proposed that noradrenalinecontaining brain systems interact with dopamine-containing brain systems in order to mediate behavior (see: Antelman and Caggiula (1977) in Verheij and Cools, 2008). Given the strong innervation of the nucleus accumbens by both noradrenergic and dopaminergic fibers, the interaction between noradrenaline and dopamine within this mesolimbic brain region has received much attention over the last 25 years (for review, see Verheij and Cools, 2008). Using microdialysis, it was shown that intraaccumbens administration of the $\alpha$-adrenoceptor agonist phenylephrine increases the extracellular levels of accumbal dopamine (Tuinstra and Cools, 2000). This increase of accumbal dopamine may, at least partly, underlie the increase in locomotor activity observed after the local administration of noradrenaline. Interestingly, the involved mesolimbic adrenoceptors have also been proposed to regulate the (individual-specific) behavioral response to environmental challenges or stressors (for review, see Verheij and Cools, 2008).

It has been found that the intra-accumbal $\alpha$-adrenoceptors that mediate the local release of dopamine are of the $\alpha-1$, but not $\alpha-2$, type (Saigusa et al, 2012). These $\alpha-1$ receptors appear to be located on both sides of the noradrenergic synapse. The finding that intra-accumbens administration of the dopamine-increasing and selective $\alpha-1$-adrenoceptor agonist phenylephrine results in a reduction of the levels of accumbal noradrenaline (Aono et al, 2007) implies that the noradrenergic axons that terminate in the nucleus accumbens are equipped with $\alpha-1$ receptors

*Correspondence: Dr MMM Verheij, Department of Cognitive Neuroscience, Division of Psychoneuropharmacology, Donders Institute for Brain, Cognition and Behaviour, Radboud University Nijmegen Medical Centre, PO Box 9I0I, 6500 HB, Nijmegen, The Netherlands, Tel: +31 24 3619565, Fax: +31 24 3541435, E-mail: M.Verheij@cns.umcn.nl

Received 14 September 2012; revised 19 September 2012; accepted 24 September 2012 (see Supplementary Figure S1). In addition, $\alpha$-1-adrenoceptor agonists can also reduce the release of accumbal dopamine without changing the accumbal levels of noradrenaline (Saigusa et al, 2012). These data indicate that the accumbal $\alpha$-1-adrenoceptors, which control the local release of dopamine, are not only presynaptically located on the noradrenergic axons, but also at the postsynaptic side (see Supplementary Figure S1). Using a double-staining procedure, Mitrano et al (2012) have recently provided direct evidence in favor of the previously reported hypothesis that these postsynaptically located $\alpha$-1-adrenoceptors of the nucleus accumbens are located on dopaminergic axons (see Tuinstra and Cools, 2000).

Given that stimulation of postsynaptically located $\alpha-1$ adrenoceptors reduces accumbal dopamine release (see above), the previously reported phenylephrine-induced dopamine increase should be ascribed to the abovementioned phenylephrine-induced reduction of noradrenaline at these postsynaptic receptors (see Supplementary Figure S1a). Consistent with a dopamine increase observed after intra-accumbens administration of the $\alpha-1$ agonist phenylephrine, Saigusa et al (2012) have recently shown that the local administration of the $\alpha-1$ antagonist prazosin decreases the levels of accumbal dopamine. According to the available data, this dopamine decrease should be ascribed to an inhibition of the accumbal $\alpha$-1-adrenoceptors that are presynaptically located on noradrenergic axons, thereby increasing the release of noradrenaline at the level of the postsynaptically located and dopamine-inhibiting $\alpha$-1-adrenoceptors of the nucleus accumbens (see Supplementary Figure S1b). This explanation is supported by the finding that the prazosin-induced accumbal dopamine decrease is indeed preceded by an increase of the extracellular levels of accumbal noradrenaline (Saigusa et al, 2012). Interestingly, whether a particular $\alpha$-1adrenergic agent acts pre- or postsynaptically depends on the (endogenous) amount of noradrenaline inside the synapse. Animals marked by low synaptic noradrenaline levels in the nucleus accumbens (eg, Sprague-Dawley, but 
not Wistar, rats) are marked by postsynaptic $\alpha$-adrenoceptors that are sensitive to agonists, whereas the presynaptic $\alpha$-adrenoceptors in these animals are sensitive to antagonists (see: Cools et al (1987) in Saigusa et al, 2012).

The $\alpha$-adrenoceptors of the nucleus accumbens are known to control the release of dopamine that is derived from reserpine-sensitive storage vesicles (Verheij and Cools, 2009). These vesicles were also found to mediate the behavioral and accumbal dopamine response to cocaine (for review, see Verheij and Cools, 2008). It was, therefore, concluded that cocaine, besides inhibiting the re-uptake of dopamine, also releases dopamine from storage vesicles (see Supplementary Figure S1c).

The above-mentioned data demonstrate that mesolimbic noradrenaline has a crucial role in modulating accumbal dopamine release and may, therefore, modulate dopaminemediated behavior. This is nicely illustrated by a study of Mitrano et al (2012), showing that the $\alpha$-1 antagonist terazosin reduces both the locomotor and accumbal dopamine response to cocaine. According to the available data, we speculate that terazosin, similar to prazosin (see above), acts at the $\alpha-1$-adrenoceptors that are presynaptically located on noradrenergic axons, thereby increasing the concentration of accumbal noradrenaline at the level of the inhibitory $\alpha-1$ receptors that are postsynaptically located on the dopaminergic axons of the nucleus accumbens (see Supplementary Figure S1d). The observed reduction in the behavioral and dopamine response to cocaine becomes understandable from the fact that this terazosin-induced noradrenaline increase results in an inhibition of the release of accumbal dopamine from the same type of storage vesicles as that from where cocaine releases accumbal dopamine (see Supplementary Figure S1d).

Given that intra-accumbens administration of the $\beta$-adrenoceptor agonists isoproterenol has also been found to increase the release of accumbal dopamine (Tuinstra and Cools, 2000; Verheij and Cools, 2009), it has been proposed that besides $\alpha$-adrenoceptor antagonists such as terazosin, $\beta$-adrenoceptor antagonists such as propranolol may also reduce the psychostimulant-induced locomotor activity (for details, see Verheij and Cools, 2008). As a final remark, we would like to recall that accumbal adrenoceptors do not only control the locomotor response to cocaine, but also more complex processes like learning and memory (for review, see Verheij and Cools, 2008). Apart from its putative therapeutic role in the treatment of psychostimulant addiction, the presented data show that noradrenergic agents may also have therapeutic effects in other diseases that are typically ascribed to a dysfunction of mesolimbic dopamine.

\section{ACKNOWLEDGEMENTS}

$\mathrm{MV}$ is supported by an ECNP research grant for young scientists.

\section{DISCLOSURE}

The authors declare no conflict of interest.

\section{REFERENCES}

Aono Y, Saigusa T, Watanabe S, Iwakami T, Mizoguchi N, Ikeda H et al (2007). Role of alpha adrenoceptors in the nucleus accumbens in the control of accumbal noradrenaline efflux: a microdialysis study with freely moving rats. J Neural Transm 114: 1135-1142.

Mitrano DA, Schroeder JP, Smith Y, Cortright JJ, Bubula N, Vezina $\mathrm{P}$ et al (2012). Alpha-1 adrenergic receptors are localized on presynaptic elements in the nucleus accumbens and regulate mesolimbic dopamine transmission. Neuropsychopharmacology 37: 2161-2172.

Saigusa T, Aono Y, Uchida T, Takada K, Verheij MM, Koshikawa N et al (2012). The alpha(1)-, but not alpha(2)-, adrenoceptor in the nucleus accumbens plays an inhibitory role upon the accumbal noradrenaline and dopamine efflux of freely moving rats. Eur J Pharmacol 688: 35-41.

Tuinstra T, Cools AR (2000). High and low responders to novelty: effects of adrenergic agents on the regulation of accumbal dopamine under challenged and non-challenged conditions. Neuroscience 99: 55-64.

Verheij MM, Cools AR (2008). Twenty years of dopamine research: Individual differences in the response of accumbal dopamine to environmental and pharmacological challenges. Eur J Pharmacol 585: 228-244.

Verheij MM, Cools AR (2009). Mesolimbic alpha-, but not betaadrenoceptors control the accumbal release of dopamine that is derived from reserpine-sensitive storage vesicles. Neuroscience 162: $1163-1173$.

Supplementary Information accompanies the paper on the Neuropsychopharmacology website (http://www.nature.com/npp) 